\title{
Healing the gap: the evolution of esophageal substitute and quality of life in patients undergoing total pharyngolaryngoesophagectomy with reconstruction
}

\author{
Tzu-Yen Chang ${ }^{1 \#}$, Chao-Chun Chang ${ }^{2 \#}$, Wen-Ruei Tang ${ }^{2}$, Wei-Ting Lee ${ }^{3}$, Yao-Chou Lee ${ }^{1}$, Wei-Li Huang², \\ Ying-Yuan Chen ${ }^{2}$, Yi-Ting Yen ${ }^{2,4}$, Jenn-Ren Hsiao ${ }^{3}$, Jung-Der Wang ${ }^{5}$, Yau-Lin Tseng ${ }^{2}$ \\ 'Division of Plastic and Reconstructive Surgery, Department of Surgery, National Cheng Kung University Hospital, Tainan 704, \\ Taiwan. \\ ${ }^{2}$ Division of Thoracic Surgery, Department of Surgery, National Cheng Kung University Hospital, Tainan 704, Taiwan. \\ ${ }^{3}$ Department of Otolaryngology, National Cheng Kung University Hospital, College of Medicine, National Cheng Kung University, \\ Tainan 704, Taiwan. \\ ${ }^{4}$ Division of Trauma and Acute Care Surgery, Department of Surgery, National Cheng Kung University Hospital, Tainan 704, \\ Taiwan. \\ ${ }^{5}$ Department of Public Health, College of Medicine, National Cheng Kung University, Tainan 704, Taiwan. \\ \#Authors contributed equally.
}

Correspondence to: Dr. Yi-Ting Yen, Division of Trauma and Acute Care Surgery, Department of Surgery, National Cheng Kung University Hospital, 138 Sheng-Li Road, Tainan 704, Taiwan. E-mail: b85401067@gmail.com

How to cite this article: Chang TY, Chang CC, Tang WR, Lee WT, Lee YC, Huang WL, Chen YY, Yen YT, Hsiao JR, Wang JD, Tseng YL. Healing the gap: the evolution of esophageal substitute and quality of life in patients undergoing total pharyngolaryngoesophagectomy with reconstruction. Plast Aesthet Res 2021;8:29. https://dx.doi.org/10.20517/23479264.2021.11

Received: 1 Feb 2021 First Decision: 6 Apr 2021 Revised: 30 Apr 2021 Accepted: 26 May 2021 First online: 9 Jun 2021

Academic Editor: Matthew Spector Copy Editor: Yue-Yue Zhang Production Editor: Yue-Yue Zhang

\begin{abstract}
Aim: Hypopharyngeal squamous cell carcinoma is reportedly one of the most aggressive primary cancers, and surgical resection continues to be the standard therapeutic choice. In patients with hypopharyngeal cancer involving the esophagus or synchronous hypopharyngeal and esophageal cancer, total pharyngolaryngoesophagectomy (TPLE) is indicated to control both malignancies at the same time. Reconstruction remains challenging with regard to the length of the substitute for the esophagus as well as the donor site morbidity. We reported our long-term follow-up and the outcome of the quality of life (QoL).
\end{abstract}


Methods: We retrospectively reviewed the records of all patients who underwent TPLE between January 2012 and December 2020. Information was collected on sex, age, surgical indications, operative time, postoperative complication, swallowing function, hospital stay, and survival. Quality of life scores were acquired by World Health Organization Quality of Life-Brief (WHOQOL-BREF) questionnaires and completed at the outpatient clinic. Gaussian kernel-smoothing was applied to estimate the dynamic changes of QoL function.

Results: A total of 40 patients undergoing oncologic pharyngolaryngoesophagectomy were enrolled in this study. There were 26 patients (65\%) undergoing gastric tube reconstruction with direct anastomosis to the oropharynx (GP group), 7 patients (18\%) undergoing additional free jejunal flap to bridge the gap between the gastric tube and oropharynx (GP-JF group), 4 patients (10\%) undergoing additional free anterolateral thigh flap to bridge the gap and resurface the neck skin (GP-ALT group), and 3 patients ( $8 \%$ ) undergoing colon interposition ( $\mathrm{Cl}$ group). The leakage rate in each group was 50\% for GP group, 29\% for GP-JF group, 50\% for GAP-ALT group, and $67 \%$ for Cl group. The mean operation time was $1010 \pm 195 \mathrm{~min}$. Although the overall leakage rate was $47.5 \%$, only $15 \%$ of the patients needed further surgical intervention. One patient (2.5\%) died with persistent leakage and pneumonia. In terms of life quality assessment, the response rate for the QoL questionnaire was 50\%. We found the overall QoL deteriorated for the first year after operation, but it gradually improved and even surpassed the patient pretreatment scores by the end of the second year after operation.

Conclusion: The gap caused by TPLE in patient, perioperative morbidity, and postoperative quality of life could be managed by the evolution of esophageal substitute, surgical techniques, perioperative wound care, and evaluation of the quality of life.

Keywords: Total pharyngolaryngoesophagectomy, negative pressure wound therapy, quality of life

\section{INTRODUCTION}

There are approximately 80,000 patients with newly diagnosed hypopharyngeal cancer and 35,000 deaths annually in the world ${ }^{[1]}$. In Taiwan, 1209 patients were newly diagnosed as having hypopharyngeal cancer in 2018, and the age-standardized incidence rate was 3.25 per 100,000 person-years. Hypopharyngeal cancer tends to be locally advanced at diagnosis because of the lack of alarming symptoms. Fewer than 50\% of hypopharyngeal cancers are confined to the hypopharynx and more than $80 \%$ of patients have stage III or IV disease at diagnosis ${ }^{[2-4]}$. Because of its late diagnosis with high rate of metastasis, the disappointing survival and functional outcome were well-known. Although organ-preserving multi-modality treatment for hypopharyngeal cancer has been prevailing, surgical resection continues to be the standard therapeutic choice $e^{[5-7]}$.

It is not uncommon for hypopharyngeal cancers to have multisite involvement and extension into adjacent mucosal areas. Postcricoid tumors usually extend through the cricoid cartilage or involve the cervical esophagus $^{[5]}$. For those patients with advanced hypopharyngeal cancers, total pharyngolaryngoesophagectomy (TPLE) with postoperative radiotherapy (RT) is usually recommended for disease eradication and prevention from local recurrence. Chemoradiation for extensive disease with organ dysfunction is likely to preserve the organ without preservation of function. Although salvaging the TPLE after a failed RT therapy always produces a high morbidity ${ }^{[6]}$, radical surgery with subsequent reconstruction and adequate adjuvant treatment are more likely to have an acceptable oncologic and functional outcome in such circumstances ${ }^{[7]}$. Routine endoscopic screening of the upper alimentary tract at the diagnosis of hypopharyngeal cancer contributes to the frequent association of hypopharyngeal cancer with esophageal cancer because of commonly shared risk factors such as cigarette smoking and alcohol consumption ${ }^{[8-11]}$. In patients with synchronous hypopharyngeal and esophageal cancer, TPLE with esophagectomy is indicated to control both malignancies at the same time. 
Reconstructing the alimentary tract after TPLE is challenging. The goal of reconstruction should be directed to single-stage surgery with low morbidity and mortality, shortened hospital stay, and rapid restoration of oral alimentation ${ }^{[12]}$. Different substitutes for the esophagus have been proposed, including gastric tube pullup or colon graft interposition, free jejunal flap transfer, and supercharge of graft with microvascular anastomosis ${ }^{[13-18]}$. Gastric pull-up is the oldest technique still in use for reconstruction after TPLE. When introduced in 1960, the procedure was characterized by its a one-stage operation, low mortality, and the patients not being worse off than before the operation ${ }^{[19]}$. However, the limited extension of gastric tube might put the anastomosis under tension if the resection margin was too high in the oropharynx or nasopharynx. Additional flap would be required to bridge this gap ${ }^{[20]}$. In this study, we report our long-term follow-up and share our experience about the management of complication.

\section{METHODS}

\section{Patient enrollment}

This retrospective study was conducted to review the medical records of patients who underwent oncologic TPLE between January 2012 and December 2020. There were 41 patients; 38 patients were reconstructed with gastric pull-up and the other 3 were reconstructed with colon interposition. Among the 38 patients with gastric pull-up, 26 patients had their gastric tube directly anastomosed to the oropharynx (GP group). Another 7 patients had free jejunal flap to bridge the gap between the gastric tube and the oropharynx (GPJF group). Another 4 patients had free anterolateral thigh flap to bridge this gap with concomitant reconstruction of the neck skin defect (GP-ALT group). One patient was reconstructed with pedicled pectoralis major myocutaneous flap to bridge this gap. Because he did not follow our algorithm, he was excluded for the following analysis.

\section{Algorithmic approach for TPLE reconstruction}

The gastric pull-up was of higher priority as the esophageal substitute than the colon interposition in our institution. Colon interposition was only considered if the stomach was previously operated. The gastric tube was pulled up via a posterior mediastinal route to reach the pharyngeal defect. The tension between the gastric tube and the resection margin at the oropharynx was evaluated. The additional free flap would be transferred by a plastic surgeon if gap between the gastric tube and the resection margin could not be resolved using Kocher maneuver. The priority of free jejunal flap was higher than anterolateral thigh flap to avoid donor site immobilization. If there was an additional neck skin defect, the anterolateral thigh flap was otherwise harvested. The surgical procedure was detailed in our previous study ${ }^{[20]}$.

\section{The evaluation of the quality of life}

Patients in this study were invited to complete the World Health Organization Quality of Life-Brief (WHOQOL-BREF) questionnaires at the outpatient clinic. The WHOQOL-BREF questionnaire is a generic psychometric instrument ${ }^{[21]}$. It contains 28 items to evaluate in the four domains: physical, psychological, social relations, and environment. Each item is scored from 1 to 5 , where a higher score indicates a better Quality of Life (QoL). By multiplying the average scores of all items in the same domain by four, a domain score is calculated. Each domain score ranges from 4 to 20 points. The validity of its use in oral cancer has been confirmed by Rasch models, confirmatory factor analysis, and Pearson correlations ${ }^{[22]}$. For each QoL assessment, the "time after treatment" was defined as the period between the operation date and the date of assessment. To estimate the dynamic changes of QoL function, Gaussian kernel smoothing was applied ${ }^{[23]}$. Namely, the estimation of the mean QoL at a particular time $t$ was the weighted average of QoL assessments, where the weights were determined by a parameter named bandwidth. The bandwidth was set at 0.1 in this study. The relevant confidence intervals for the mean function estimations of QoL was constructed by a bootstrap approach. Each patient was the unit used for bootstrapping. At each time point, a $95 \%$ confidence internal was constructed using the 2.5 and 97.5 percentiles of 76 mean QoL estimated 
from bootstrapping. This study was approved by our institutional review board (A-ER-108-525).

\section{Statistical analysis}

For the numerical variables, the data are mean \pm standard deviation and were analyzed using the Wilcoxon test because the normality tests (Kolmogorov-Smirnov test and Shapiro-Wilk test) rejected the null hypothesis and concluded that these data did not come from normal distribution. For the categorical variables, the data were analyzed using Pearson's $\chi^{2}$ test or Fisher's Exact test, as appropriate. Significance was set at $P<0.05$ (two-tailed). Statistical software, MedCalc (version 19.5), was used to perform statistical analysis of data and results.

\section{RESULTS}

A total of 40 patients undergoing oncologic TPLE were further analyzed [Table 1 and 2]. All but one was male. The indication for 18 patients (45\%) was hypopharyngeal or laryngeal cancer with esophageal extension, while indication for the rest of the 22 patients (55\%) was synchronous hypopharyngeal or laryngeal cancer and esophageal cancer. Twenty-four patients (60\%) were primarily treated with surgery, and the rest of the 16 patients ( $40 \%$ ) were treated with salvage operation. The mean operation time was 1010 $\pm 195 \mathrm{~min}$, and the mean duration of hospitalization was $41 \pm 31$ days. The distribution of hypopharyngeal cancer stages was the following: 1 (2.5\%) was stage I, 2 (5\%) were stage II, 8 (20\%) were stage III, 23 (57.5\%) were stage IVa, and $6(15 \%)$ were stage IVb. The overall survival time were $45 \pm 7$ months [Figure 1]. The mean survival time was $43 \pm 16$ months for stage III, $50 \pm 9$ months for stage IVa, and $12 \pm 3$ months for stage IVb [Figure 2]. There was no difference in survival time between those primarily treated with surgery (mean 42 months; 95\%CI: 27-58 months) and those treated with salvage operation (mean 41 months; 95\%CI: 19-63 months) $(P=0.940)$ [Figure 3].

Thirty-seven patients were reconstructed using gastric pull-up [Table 2]. Among them, 26 patients (70\%) had the gastric tube directly anastomosed to the oropharynx (GP group). Another 7 patients (19\%) used additional free jejunal flap to bridge the gap between gastric tube and oropharynx (GP-JF group). The rest 4 patients (11\%) had additional free anterolateral thigh flap to bridge the gap and resurface the neck skin (GPALT group). The operation time was significantly lower in the GP group (918 $\pm 152 \mathrm{~min}$ ) than the GP-JF $(1166 \pm 164 \mathrm{~min})$ and GP-ALT group $(1166 \pm 90 \mathrm{~min})(P<0.001)$. Successful swallowing was achieved by more than $70 \%$ patients in gastric pull-up group, and the GF-JF group had the highest rate as $85 \%(6 / 7)$ patients swallowed successfully. Other than gastric pull-up, we had 3 patients who underwent colon interposition (CI group). However, only one patient in the CI group swallowed successfully in the end.

Regarding surgical complications, anastomosis leakage occurred in 19 patients (47.5\%). The leakage rate was as high as $50 \%(13 / 26)$ in the GP group, but as low as $29 \%(2 / 7)$ in the GP-JF group, but there was no statistical significance $(P=0.413)$. However, most of the leakage was managed conservatively. Two patients in the GP group had partial necrosis of the gastric tube. One was salvaged by deltopectoral flap, and the other was salvaged by pectoralis major flap. In the GP-JF group, there was one jejunal flap partial necrosis with leakage, salvaged by deltopectoral flap. In the GP-ALT group, 2 patients (50\%) had leakage from anastomosis between flap and gastric tube. One was salvaged by simple closure, and the other one closed with pectoralis major flap. In the CI group, 2 patients (67\%) had colon graft necrosis. One was salvaged by pectoralis major flap, and the other one had persistent leakage even though pectoralis major and deltopectoral flaps were used. Unfortunately, the patient died of pneumonia as the only intrahospital mortality $(2.5 \%, 1 / 40)$ in the study cohort. One patient in the GP group and one in the CI group developed tracheoesophageal fistula at the outpatient clinic. The former was salvaged by tracheal stent, and the latter, salvaged by tracheal stent along with deltopectoral flap. 
Table 1. Characteristics of patients underwent total laryngopharyngoesophagectomy and reconstruction

\begin{tabular}{|c|c|c|c|c|c|c|c|c|}
\hline Case & Age & Sex & Dx & Recon. & Complication & Oral intake & Follow up & Outcome \\
\hline 1 & 47 & $\mathrm{~F}$ & $\mathrm{HCa}-\mathrm{ECa}$ & GP & Leakage & Failed & 13 & Bleeding; died \\
\hline 2 & 72 & M & $\mathrm{HCa}$ & GP & & POD 15 & 23 & Renal failure; died \\
\hline 3 & 53 & M & $\mathrm{HCa}$ & GP & & POD 15 & 12 & Pneumonia; died \\
\hline 4 & 59 & M & $\mathrm{HCa}$ & GP & & POD 15 & 107 & NER; survived \\
\hline 5 & 50 & M & $\mathrm{HCa}$ & GP & Leakage & Failed & 15 & Pneumonia; died \\
\hline 6 & 51 & M & $\mathrm{HCa}$ & GP & Leakage & POD 50 & 14 & Pneumonia; died \\
\hline 7 & 62 & M & $\mathrm{HCa}$ & GP & & POD 30 & 5 & Pneumonia; died \\
\hline 8 & 68 & M & $\mathrm{HCa}$ & GP & Leakage & POD 54 & 90 & NER; survived \\
\hline 9 & 53 & M & $\mathrm{HCa}-\mathrm{ECa}$ & GP & Leakage, stricture & POD 39 & 86 & NER; survived \\
\hline 10 & 48 & M & $\mathrm{HCa}$ & GP & & POD 19 & 83 & NER; survived \\
\hline 11 & 56 & M & $\mathrm{HCa}$ & GP & Leakage & Failed & 5 & Bleeding; died \\
\hline 12 & 55 & M & $\mathrm{HCa}-\mathrm{ECa}-\mathrm{TCa}$ & GP & Leakage & POD 180 & 80 & NER; survived \\
\hline 13 & 43 & M & $\mathrm{HCa}-\mathrm{ECa}$ & GP & & POD 31 & 15 & UTI; died \\
\hline 14 & 63 & M & $\mathrm{HCa}$ & GP & Leakage & Failed & 3 & Pneumonia; died \\
\hline 15 & 53 & M & $\mathrm{HCa}-\mathrm{ECa}$ & GP & & POD 23 & 53 & Recurrence; died \\
\hline 16 & 51 & M & $\mathrm{HCa}-\mathrm{ECa}$ & GP & & POD 20 & 19 & Recurrence; died \\
\hline 17 & 60 & M & $\mathrm{HCa}$ & GP & T-E fistula & POD 25 & 20 & NER; survived \\
\hline 18 & 39 & M & $\mathrm{HCa}-\mathrm{ECa}$ & GP & Leakage, stricture & POD 47 & 18 & Liver mets; died \\
\hline 19 & 64 & M & LCa-ECa & GP & Leakage & POD 41 & 38 & NER; survived \\
\hline 20 & 63 & M & $\mathrm{LCa}-\mathrm{ECa}$ & GP & Gastric tube necrosis; PM salvage & Failed & 27 & NER; survived \\
\hline 21 & 57 & M & $\mathrm{LCa}-\mathrm{ECa}$ & GP & & POD 60 & 24 & NER; survived \\
\hline 22 & 46 & M & LCa-ECa & GP & Leakage & Failed & 7 & Pneumonia; died \\
\hline 23 & 52 & M & $\mathrm{HCa}-\mathrm{ECa}$ & GP & & POD 17 & 7 & Bleeding; died \\
\hline 24 & 52 & M & $\mathrm{HCa}-\mathrm{ECa}$ & GP & Leakage, DP salvage & POD 90 & 17 & NER; survived \\
\hline 25 & 66 & M & $\mathrm{HCa}-\mathrm{ECa}-\mathrm{TCa}$ & GP & Chylous leakage & Failed & 5 & NER; survived \\
\hline 26 & 79 & M & $\mathrm{HCa}$ & GP & & POD 37 & 7 & NER; survived \\
\hline 27 & 70 & M & $\mathrm{HCa}-\mathrm{ECa}$ & $\mathrm{Cl}$ & Leakage; PM, DP salvage & Failed & 3 & Pneumonia; died \\
\hline 28 & 50 & M & LCa-ECa & $\mathrm{Cl}$ & Empyema, chylo, T-E fistula & POD 58 & 81 & NER; survived \\
\hline 29 & 61 & M & $\mathrm{HCa}-\mathrm{ECa}$ & $\mathrm{Cl}$ & Leakage, PM salvage & Failed & 4 & NER; survived \\
\hline 30 & 59 & M & $\mathrm{HCa}-\mathrm{ECa}$ & GP-JF & & POD 76 & 12 & Pneumonia; died \\
\hline 31 & 45 & M & HCa-ECa-MFCa & GP-JF & & POD 28 & 29 & Died \\
\hline 32 & 41 & M & $\mathrm{HCa}$ & GP-JF & & POD 38 & 8 & Pneumonia; died \\
\hline 33 & 57 & M & $\mathrm{HCa}$ & GP-JF & Leakage & Failed & 7 & Lung mets; died \\
\hline 34 & 46 & M & $\mathrm{HCa}-\mathrm{ECa}$ & GP-JF & & POD 28 & 20 & Bleeding; died \\
\hline 35 & 58 & M & $\mathrm{HCa}-\mathrm{ECa}-\mathrm{PCa}$ & GP-JF & & POD 28 & 33 & Recurrence; died \\
\hline 36 & 58 & M & $\mathrm{HCa}$ & GP-JF & Leakage; DP salvage & POD 62 & 22 & LN, bone mets; died \\
\hline 37 & 55 & M & $\mathrm{LCa}$ & GP-ALT & & POD 30 & 106 & NER; survived \\
\hline 38 & 51 & M & $\mathrm{HCa}-\mathrm{ECa}$ & GP-ALT & Leakage & POD 36 & 73 & Died \\
\hline 39 & 55 & M & $\mathrm{HCa}$ & GP-ALT & & POD 42 & 9 & Bleeding; died \\
\hline 40 & 56 & M & $\mathrm{HCa}$ & GP-ALT & Leakage; PM salvage & Failed & 7 & NER; survived \\
\hline
\end{tabular}

HCa: Hypopharyngeal cancer; ECa: esophageal cancer; LCa: laryngeal cancer; TCa: tongue cancer; MFCa: mouth floor camcer; PCa: palatal cancer; Recon.: reconstruction type; GP: pure gastric pull-up; Cl: colon interposition; GP-JF: gastric pull-up with free jejunal flap; GP-ALT: gastric pull-up with free anterolateral thigh flap; GP-PM: gastric pull-up with pectoralis major myocutaneous flap; POD: postoperative day; NER: no evidence of recurrence; Mets: metastasis; LN: Iymph node; UTI: urinary tract infection; Chylo: chylothorax; PM: pectoralis major myocutaneous flap; DP: deltopectoral flap.

Twenty patients (50\%) had been assessed for their QoL at least one time [Figure 4]. The overall QoL and general health (Q1) deteriorated for the first year after the index operation. However, it gradually improved 
Table 2. Patients underwent different reconstruction after total laryngopharyngoesophagectomy

\begin{tabular}{|c|c|c|c|c|c|}
\hline & Total & GP & GP-JF & GP-ALT & Cl \\
\hline$n$ & 40 & 26 & 7 & 4 & 3 \\
\hline $\operatorname{Sex}, M / F$ & $39 / 1$ & $25 / 1$ & $7 / 0$ & $4 / 0$ & $3 / 0$ \\
\hline Age, y/o (SD) & $55.6(8.5)$ & $56.2(9.2)$ & $52.0(7.7)$ & $54.3(2.2)$ & $60.3(10.0)$ \\
\hline \multicolumn{6}{|l|}{ Indication } \\
\hline Esophageal extension, $n$ & 18 & 12 & 3 & 3 & 0 \\
\hline Double cancer, $n$ & 22 & 14 & 4 & 1 & 3 \\
\hline Primary, $n$ & 24 & 17 & 3 & 3 & 1 \\
\hline Salvage, $n$ & 16 & 9 & 4 & 1 & 2 \\
\hline Operation time, $\min$ & $1010(195)$ & $918(152)$ & $1166(164)$ & $1166(90)$ & $1230(207)$ \\
\hline Leakage, $n(\%)$ & $19(47.5)$ & $13(50)$ & $2(28.6)$ & $2(50)$ & $2(66.7)$ \\
\hline Stricture, $n(\%)$ & $2(6.9)$ & $2(10.5)$ & 0 & 0 & 0 \\
\hline Successful swallowing, $n(\%)$ & $29(72.5)$ & $20(73.1)$ & $6(85.7)$ & $3(75.0)$ & $1(33.3)$ \\
\hline Hospitalization length, mean day (SD) & $41(31)$ & $29(10)$ & $51(44)$ & $81(58)$ & $70(5)$ \\
\hline Follow-up time, mean months (SD) & $30(32)$ & $31(31)$ & $19(10)$ & $49(49)$ & $29(45)$ \\
\hline
\end{tabular}

GP: Pure gastric pull-up; Cl: colon interposition; GP-JF: gastric pull-up with free jejunal flap; GP-ALT: gastric pull-up with free anterolateral thigh flap; SD: standard deviation.

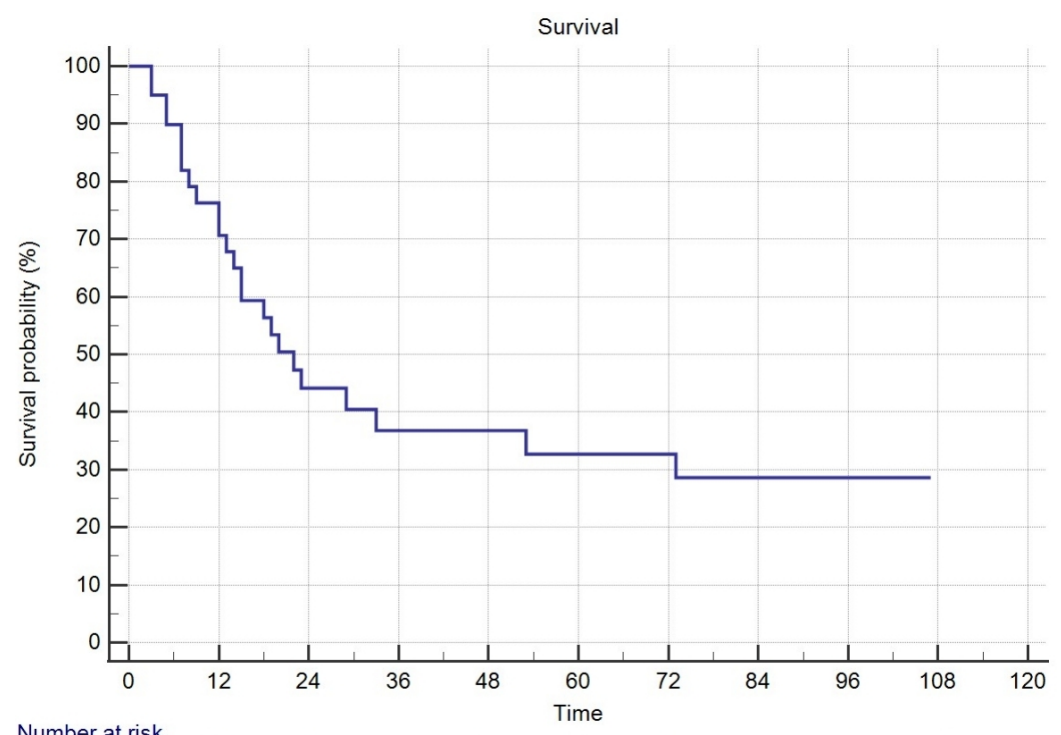

$\begin{array}{cccccccccc}\text { Number at risk } & 25 & 13 & 10 & 9 & 8 & 8 & 4 & 2 & 0\end{array}$

Figure 1. The overall survival time for the entire cohort were $45 \pm 7$ months. 


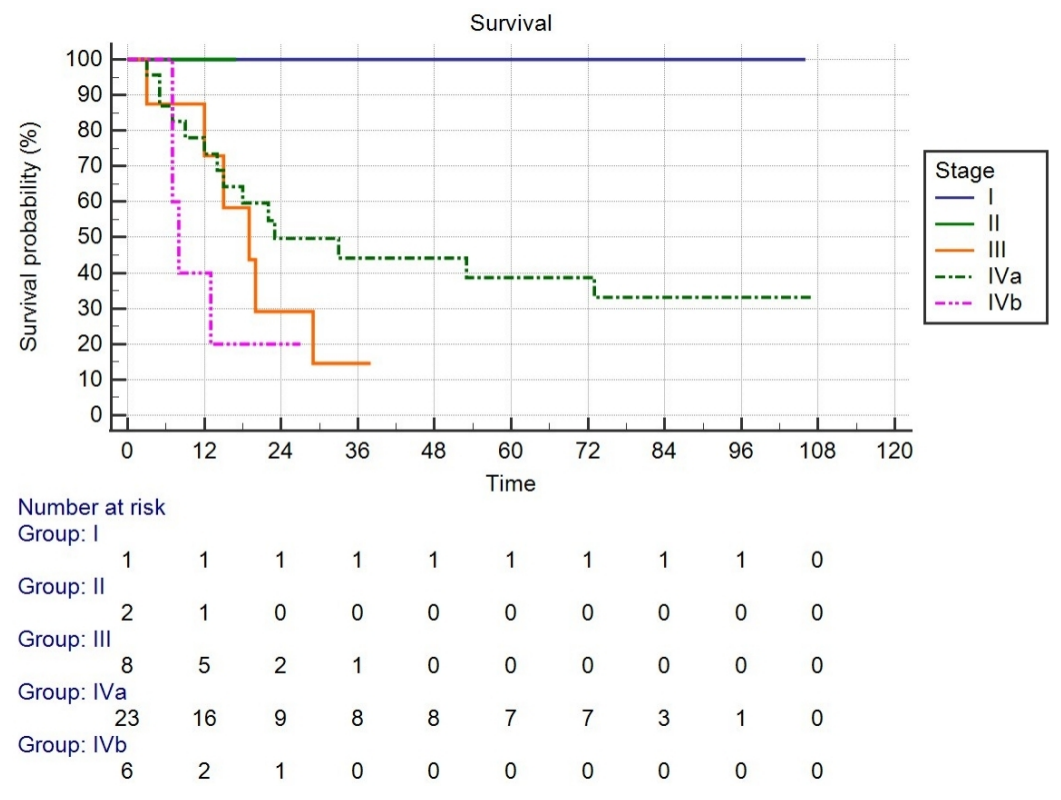

Figure 2. The hypopharyngeal cancer stage distribution was as follows: 1 (2.5\%) was stage I, 2 (5\%) were stage II, 8 (20\%) were stage III, $23(57.5 \%)$ were stage IVa, and 6 (15\%) were stage IVb. The mean survival time was $43 \pm 16$ months for stage III, $50 \pm 9$ months for stage IVa, $12 \pm 3$ months for stage IVb.

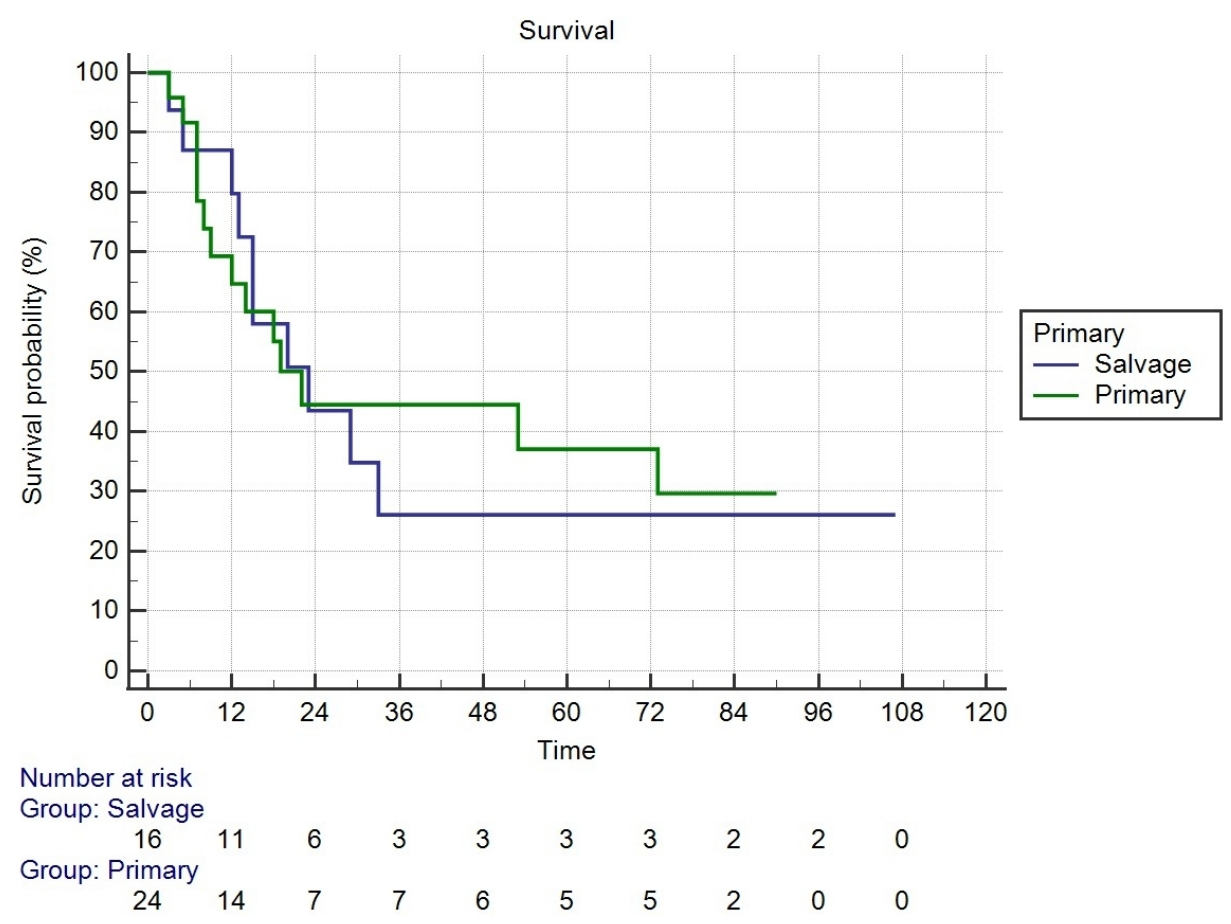

Figure 3. There was no difference between those primarily treated with surgery (mean 42 months; $95 \% \mathrm{Cl}$ : $27-58$ months) and those treated with salvage operation (mean 41 months; 95\%Cl: 19-63 months) $(P=0.9396)$.

and even surpassed their pretreatment QoL score by the end of the second year after the index operation. Since then, the QoL reached a constant level. Similar curve could be found in pain and discomfort (Q3), activities of daily living (Q17), work capacity (Q18), negative feeling (Q26), and sexual activity (Q21). Sleep 

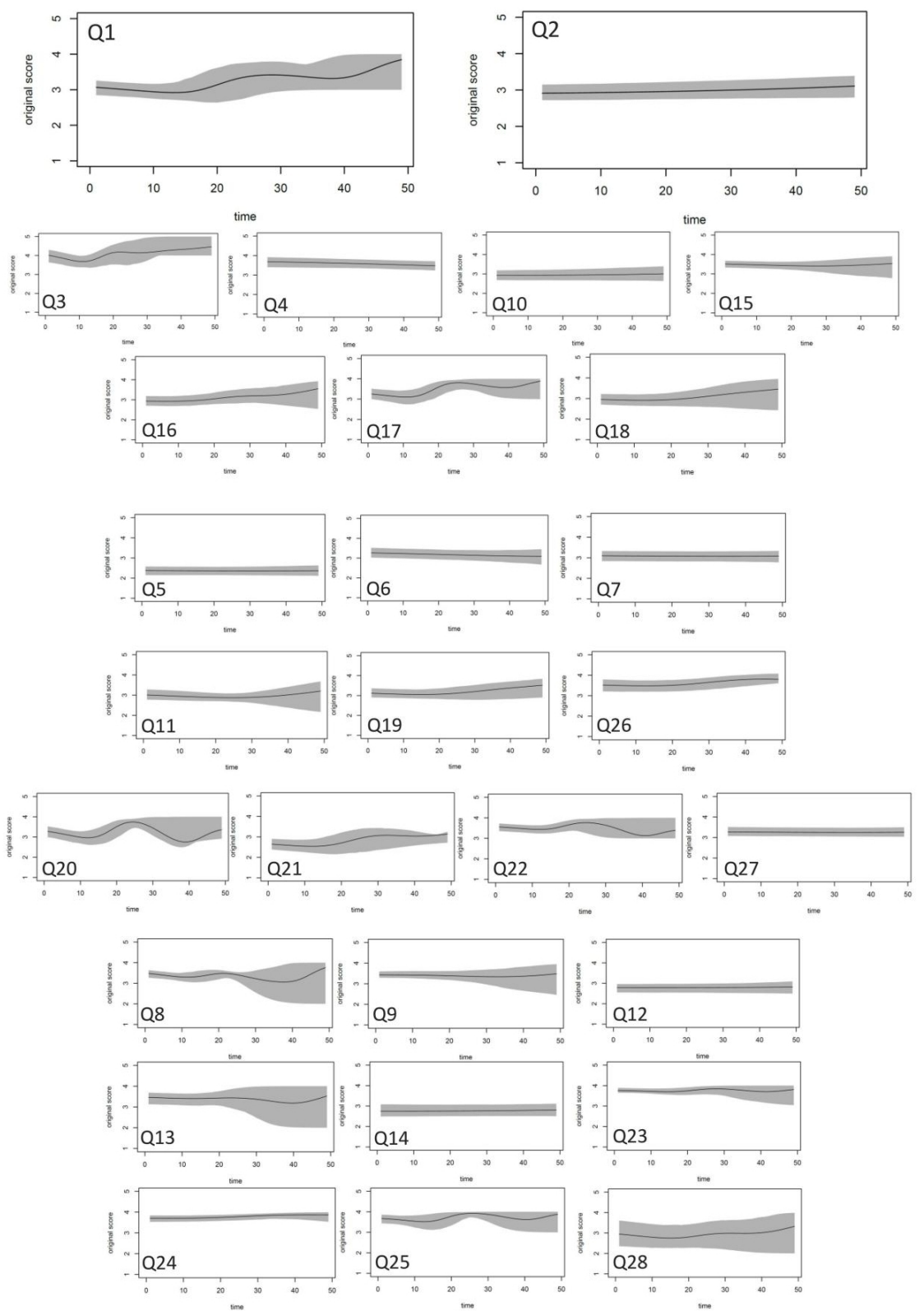

Figure 4. The World Health Organization Quality of Life - Brief (WHOQOL-BREF) questionnaire contains 28 items to evaluate four domains. The Q1 and Q2 represent overall QoL and general health. The physical domain consists of pain and discomfort (Q3), dependence on medical substances and medical aids (Q4), energy and fatigue (Q10), mobility (Q15), sleep and rest (Q16), activities of daily living (Q17), and work capacity (Q18). The psychologic domain consists of positive feelings (Q5), spirituality/religion/personal beliefs (Q6), thinking/learning/memory/concentration (Q7), bodily image and appearance (Q11), self-esteem (Q19), and negative feelings (Q26). The social relations domain consists of personal relationships (Q20), sexual activity (21), practical social support (Q22), and being respected/accepted (Q27). The environment domain consists of freedom, physical safety, and security (Q8), physical environment (Q9), financial resources (Q12), opportunities for acquiring new information and skills (Q13), participation in and opportunities for recreation/leisure activities (Q14), home environment (Q23), health and social care: accessibility and quality (Q24), transport (Q25), and eating/food (Q28). The overall QoL and general health (Q1) deteriorated in the first year after operation. However, it gradually improved and even surpassed their pretreatment QoL score by the end of the second year after operation. Since then, the QoL reached a constant level. Similar curve could be found in pain and discomfort (Q3), activities of daily living (Q17), work capacity (Q18), negative feeling (Q26), and sexual activity (Q21). Otherwise, sleep and rest (Q16) and self-esteem (Q19) slowly improved during follow up after surgery. However, substances and medical aids (Q4), and spirituality/religion/personal beliefs (Q6), deteriorated after surgery and did not recovered in the end. Interestingly, some QoL, like personal relationships (Q20), practical social support (Q22), freedom/physical safety/security (Q8), transport (Q25), and eating/food (Q28), showed regular oscillation after surgery with year. 
and rest (Q16) and self-esteem (Q19) slowly improved during the follow up visits after surgery. However, substances and medical aids (Q4) and spirituality/religion/personal beliefs (Q6) deteriorated after surgery and did not improve in the end of the follow up. Interestingly, some QoL, like personal relationships (Q20), practical social support (Q22), freedom/physical safety/security (Q8), transport (Q25), and eating/food (Q28), demonstrated regular annual oscillation after surgery.

\section{DISCUSSION}

\section{Rationale for esophageal substitute}

The surgical option of esophageal substitute varies and depends on the individualized basis of the surgeon's preference and the patient's physical feasibility. Gastric tube pull-up has been advocated and proved as an effective measure for reconstruction of the alimentary tract after TPLE, but it comes with a reportedly high rate of anastomotic leakage and surgical complications ${ }^{[24-26]}$. The combination with free jejunal flap interposition overcomes the gap between the gastric tube and the resection margin. Since Asamura et al. ${ }^{[17]}$ documented the first case, it has been reported to decrease the tension of anastomosis and the leakage rate $^{[27]}$. Colon graft interposition is another choice for alimentary tract reconstruction especially in patients who were previously operated on for peptic ulcer. Vascular supply, however, greatly impacts on the postoperative graft viability and patient outcome. It has been reported that the colon is the most preferred and safest organ for esophageal reconstruction, whereas the stomach is a vascular and muscular organ with lower risk of ischemia ${ }^{[28]}$. Moreover, the study on the QoL after esophagectomy reported that the functional results with a colon interposition were better than those with a gastric tube ${ }^{[29]}$.

Reconstruction of the alimentary tract using visceral organs avoids strict postoperative immobilization of the extremity and enables early ambulation and rehabilitation, compared to the use of the free flap harvested from the extremities. For the long-segment gap between the gastric tube and resection margin, the redundancy of free jejunal flap should be taken into consideration to avoid swallowing difficulty. Nonetheless, the superior healing ability of jejunal mucosa minimizes the incidence of anastomotic leakage and complications requiring further surgery ${ }^{[30]}$.

Occasionally, cancer invasion of the skin results in skin defect in the neck region that will expose the great vessels postoperatively. The use of a free anterolateral thigh flap to reconstruct the gap between the gastric tube and the oropharynx and the coexisting neck skin defect is optimal. We proposed the "butterfly" design, which made the anastomosis externalized at the body surface ${ }^{[20]}$. By this design, it is easy to detect any leakage from the anastomosis, and it is also safe because the leakage would not accumulate in the neck. Therefore, we provided an algorithmic approach to reconstruct the defect of the TPLE. Gastric pull-up is simple and effective to restore alimentary continuity. When tension existed between the gastric tube and the oropharynx, an additional free jejunal flap is vital to solve the problem. If there is concomitant skin defect in the neck, the combined use of gastric pull-up and free anterolateral thigh flap would be ideal. The colon interposition should be reserved for patients with previous gastric surgery or disease. Alternatively, a pedunculated gastric conduit using a Roux-en-Y anastomosis precludes the need for a second flap, but it requires an additional anastomosis in the abdomen ${ }^{[30,31]}$. Additional use of pectoralis myocutaneous flap other than gastric pull-up is also optimal if the patient is unsuitable for microvascular surgery ${ }^{[32]}$.

\section{Negative pressure wound therapy}

A substantial proportion of patients with hypopharyngeal cancer undergoing TPLE were previously irradiated ${ }^{[3,34]}$, portending the risk of anastomotic leakage and vascular fistula secondary to soiling of saliva around the anastomosis and secretion of digestive tract ${ }^{[35]}$. Anastomotic leakage constitutes a major proportion of surgical complications in patients undergoing TPLE for hypopharyngeal cancer ${ }^{[36-38]}$. The 
accumulated saliva and localized abscess resulted in vascular erosion and even aspiration pneumonia if the abscess drained out of the wound into the permanent tracheostomy. The continuous vacuum-assisted drainage adopted right after surgery evacuated the saliva as soon as it leaked, forming a controlled enterocutaneous fistula. The continuous negative pressure was created using suction tube embedded in the Penrose drain and connected to the bedside wall suction unit [Figure 5]. The application of vacuum-assisted drain decreased the frequency of wound dressing change, facilitated the postoperative wound care, reduced the risk of saliva soiling, and enhanced early bedside mobilization.

Anastomosis leakage of the intestinal mucosa in abdominal surgery was initially managed with drainag ${ }^{[3,40]}$. Because of the positive pressure in the abdomen, intra-abdominal abscess transformed into enterocutaneous fistula after the drainage ${ }^{[41-43]}$. The anatomical difference with the lack of positive pressure in the neck area resulted in abscess formation secondary to the anastomotic leakage despite of adequate drainage. The negative pressure in the conventional negative-pressure drainage device gradually decreased as the drainage amount in the device increases ${ }^{[4]}$. The continuous negative pressure in the vacuum-assisted drainage created steady suction pressure, evacuated the leakage, and provoked adhesion around the anastomosis ${ }^{[45,46]}$. In patients with microvascular anastomosis, a conventional negative-pressure drain was placed around the pedicle instead. Nonetheless, a negative-pressure drain could still be applied on the other side.

\section{Outcome evaluation of head and neck cancer reconstruction: QoL}

Defect of the head and neck poses a significant functional and emotional impact. Head and neck reconstruction should focus not only on wound resurfacing, but also function restoration. It is fundamental to evaluate the outcome with respect to both the clinical aspect and the QoL perceived by patients. In 2009, the United States' Food and Drug Administration approved the patient-reported outcome as a validated measure of treatment benefit or risk in clinical trials ${ }^{[47]}$. It is widely used in the fields of breast cancer and lung cancer ${ }^{[48,49]}$. Dissecting the outcome evaluation like the QoL in the head and neck reconstruction helps us acquire what is optimal in some inconclusive reconstruction.

Although our patient numbers were still not large enough to test our thesis in detail or subgroup analysis, the dynamic changes of the QoL in patients undergoing TPLE still gave us some clinical implications. The deterioration of the overall QoL and general health right after surgery for one year may result from surgical complication, adjuvant therapy, and the frustration from the sense of well-being. This period seemed longer than those of other head and neck patients, which usually lasts for 3 to 6 months ${ }^{[50]}$. Higher rate of surgical complication might also be responsible for this difference. However, the QoL recovered or even surpassed the pretreatment scores during the second year. This result supported the proof of the concept that surgical resection remained the standard therapeutic choice.

Two factors of the QoL constantly deteriorated after surgery despite addressing the patients' complications and having the patients acclimate to this disease. One factor is "How much medical treatment the patient needs to function in his or her daily life"? We might attribute it to the assistive device for voice rehabilitation or tube feeding due to failed swallowing function. The other factor is "How meaningful is life to the patient"? The results of these two factors are quite discouraging; however, we must factor in the significance of the psychological aspects that these patients face in such a devastating disease.

The response rate for the QoL was 50\% in this study. Patients responded in the outpatient clinic, which implied that they were the patients with the better outcome than those who did not respond. The representation for the outcome of the QoL might be biased and overestimated. Nonetheless, the patients' 


\section{Enhanced tissue adhesion}

forming a controlled enterocutaneous tract

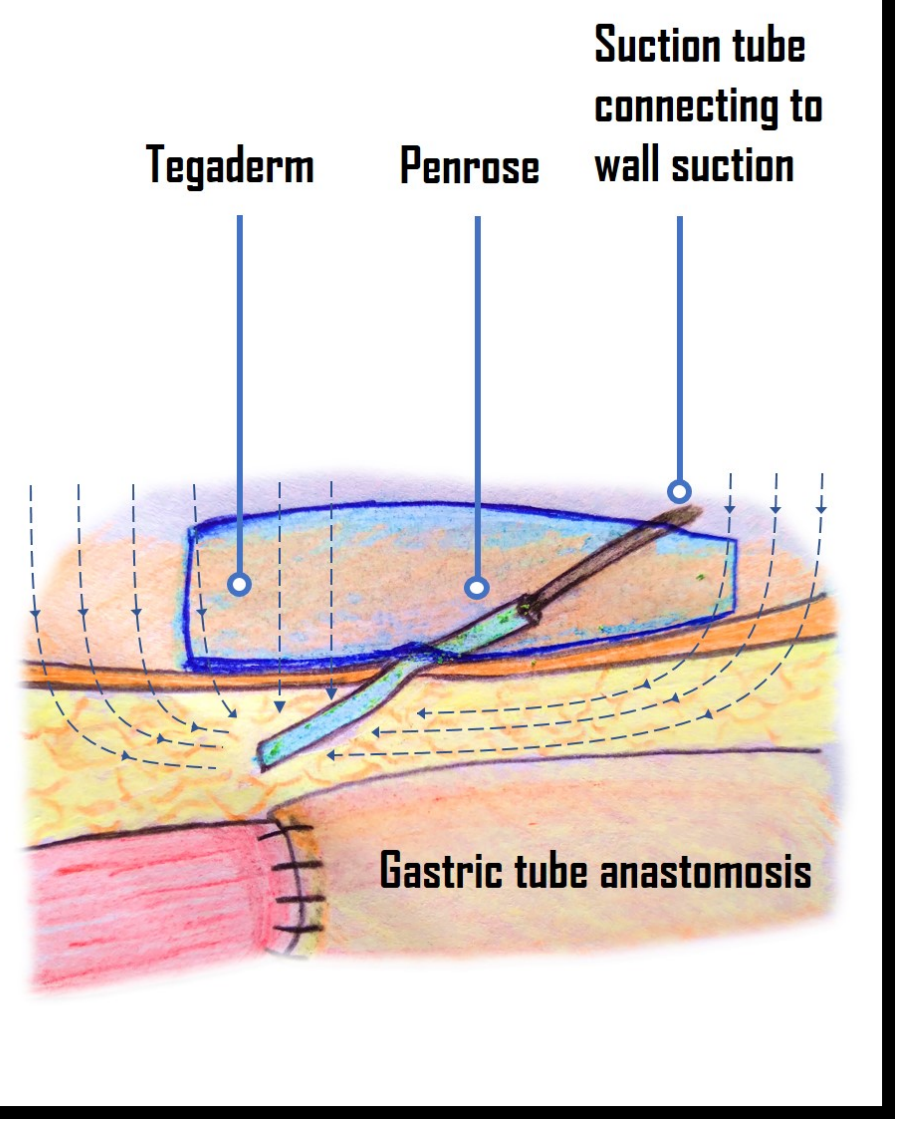

Figure 5. The negative pressure wound therapy (NPWT) facilitates formation of a controlled enterocutaneous fistula and avoids saliva soiling with subsequent abscess formation. Continuous and steady negative pressure was applied with connection of the suction tube to the bedside wall suction unit.

reported outcome was important and reflected their psychological well-being and resilience.

In summary, the evolution of esophageal substitute follows the improvement of surgical techniques and perioperative care. Different measures have been taken to heal the gap between the gastric tube pull-up and the resection margin in the oropharynx or even the nasopharynx. The postoperative QoL is becoming an issue worthy of serious consideration as long as complications after TPLE could be successfully tackled. The gap caused by TPLE in patients, perioperative morbidity, and postoperative QoL could be managed by the evolution of esophageal substitute, surgical techniques, perioperative wound care, and QoL evaluation.

\section{DECLARATIONS}

Acknowledgments

We are sincerely grateful to Dr. Wen-Ruei Tang for the originality of legendary illustration of Figure 5 . We 
declare that there is no outside funding for this article, and there is no conflict of interest with any companies or organizations whose products or services may be discussed in this article.

\section{Authors' contributions}

Data acquisition, data analysis, interpretation, study design, and manuscript writing: Chang TY, Chang CC, Yen YT

Figure 5 illustration and manuscript writing: Tang WR

Statistical consultation and quality of life interpretation: Wang JD

Administrative and technical support: Hsiao JR, Tseng YL

Surgical consultation: Lee WT, Lee YC, Huang WL, Chen YY

\section{Availability of data and materials}

Not applicable.

\section{Financial support and sponsorship}

None.

\section{Conflicts of interest}

All authors declared that there are no conflicts of interest.

\section{Ethical approval and consent to participate}

This study has been approved by Institutional Review Board of National Cheng Kung University Hospital (A-ER-108-525).

\section{Consent for publication}

Not applicable.

\section{Copyright}

(c) The Author(s) 2021.

\section{REFERENCES}

1. Bray F, Ferlay J, Soerjomataram I, Siegel RL, Torre LA, Jemal A. Global cancer statistics 2018: GLOBOCAN estimates of incidence and mortality worldwide for 36 cancers in 185 countries. CA Cancer J Clin 2018;68:394-424. DOI PubMed

2. Kuo P, Chen MM, Decker RH, Yarbrough WG, Judson BL. Hypopharyngeal cancer incidence, treatment, and survival: temporal trends in the United States. Laryngoscope 2014;124:2064-9. DOI PubMed

3. Uzcudun AE, Bravo Fernández P, Sánchez JJ, et al. Clinical features of pharyngeal cancer: a retrospective study of 258 consecutive patients. J Laryngol Otol 2001;115:112-8. DOI PubMed

4. Spector JG, Sessions DG, Haughey BH, et al. Delayed regional metastases, distant metastases, and second primary malignancies in squamous cell carcinomas of the larynx and hypopharynx. Laryngoscope 2001;111:1079-87. DOI PubMed

5. Harrison DF. Pathology of hypopharyngeal cancer in relation to surgical management. J Laryngol Otol 1970;84:349-67. DOI PubMed

6. Affleck DG, Karwande SV, Bull DA, Haller JR, Stringham JC, Davis R. Functional outcome and survival after pharyngolaryngoesophagectomy for cancer. Am J Surg 2000;180:546-50. DOI PubMed

7. Takes RP, Strojan P, Silver CE, et al; International Head and Neck Scientific Group. Current trends in initial management of hypopharyngeal cancer: the declining use of open surgery. Head Neck 2012;34:270-81. DOI PubMed

8. Bagnardi V, Rota M, Botteri E, et al. Alcohol consumption and site-specific cancer risk: a comprehensive dose-response meta-analysis. Br J Cancer 2015;112:580-93. DOI PubMed PMC

9. Viner B, Barberio AM, Haig TR, Friedenreich CM, Brenner DR. The individual and combined effects of alcohol consumption and cigarette smoking on site-specific cancer risk in a prospective cohort of 26,607 adults: results from Alberta's Tomorrow Project. Cancer Causes Control 2019;30:1313-26. DOI PubMed

10. Hung S, Tsai M, Liu T, Lin H, Chung S, Yendamuri S. Routine endoscopy for esophageal cancer is suggestive for patients with oral, oropharyngeal and hypopharyngeal cancer. PLoS One 2013;8:e72097. DOI PubMed PMC 
11. Wang WL, Wang CP, Wang HP, et al. The benefit of pretreatment esophageal screening with image-enhanced endoscopy on the survival of patients with hypopharyngeal cancer. Oral Oncol 2013;49:808-13. DOI PubMed

12. Surkin MI, Lawson W, Biller HF. Analysis of the methods of pharyngoesophageal reconstruction. Head Neck Surg 1984;6:953-70. DOI PubMed

13. Yamamoto Y, Sasaki S, Furukawa H, Okushiba S, Ohno K, Sugihara T. Microsurgical reconstruction of the digestive tract following pharyngolaryngectomy and total esophagectomy. Ann Plast Surg 1998;41:22-6; discussion 26. DOI PubMed

14. Nakatsuka T, Harii K, Ebihara S, et al. Free colon transfer: a versatile method for reconstruction of pharyngoesophageal defects with a large pharyngostoma. Ann Plast Surg 1996;37:596-603. PubMed

15. Matsubara T, Ueda M, Nakajima T, Kamata S, Kawabata K. Elongated stomach roll with vascular microanastomosis for reconstruction of the esophagus after pharyngolaryngoesophagectomy. J Am Coll Surg 1995;180:613-5. PubMed

16. Omura K, Misaki T, Watanabe Y, Urayama H, Hashimoto T, Matsu T. Reconstruction with free jejunal autograft after pharyngolaryngoesophagectomy. Ann Thorac Surg 1994;57:112-7. DOI PubMed

17. Asamura H, Kato H, Watanabe H, Tachimori Y, Ebihara S, Harii K. Combined gastric pull-up and microvascular jejunal transfer procedure after pharyngolaryngoesophagectomy. Ann Thorac Surg 1989;48:423-5. DOI PubMed

18. Tabah RJ, Flynn MB, Acland RD, Banis JC, Jr. Microvascular free tissue transfer in head and neck and esophageal surgery. Am J Surg 1984;148:498-504. DOI PubMed

19. Ong GB, Lee TC. Pharyngogastric anastomosis after oesophago-pharyngectomy for carcinoma of the hypopharynx and cervical oesophagus. Br J Surg 1960;48:193-200. DOI PubMed

20. Chang TY, Hsiao JR, Lee WT, et al. Esophageal reconstruction after oncological total laryngopharyngoesophagectomy: algorithmic approach. Microsurgery 2019;39:6-13. DOI PubMed

21. Yao G, Chung CW, Yu CF, Wang JD. Development and verification of validity and reliability of the WHOQOL-BREF Taiwan version. J Formos Med Assoc 2002;101:342-51. PubMed

22. Lin CY, Hwang JS, Wang WC, et al. Psychometric evaluation of the WHOQOL-BREF, Taiwan version, across five kinds of Taiwanese cancer survivors: Rasch analysis and confirmatory factor analysis. J Formos Med Assoc 2019;118:215-22. DOI PubMed

23. Hwang J, Wang J. Integrating health profile with survival for quality of life assessment. Qual Life Res 2004;13:1-10. DOI PubMed

24. Dai Z, He Q, Pan B, Liu L, Zhou D. Postoperative complication assessments of different reconstruction procedures after total pharyngolaryngoesophagectomy: tubular gastric pull-up versus whole gastric pull-up. Am Surg 2018;84:1927-31. PubMed

25. Sugiyama N, Takao S, Suzuki E, Kimata Y. Risk factors for wound complications in head and neck reconstruction: 773 free jejunal reconstruction procedures after total pharyngolaryngoesophagectomy. Head Neck 2017;39:2057-69. DOI PubMed

26. Miyata H, Sugimura K, Motoori M, et al. Clinical assessment of reconstruction involving gastric pull-up combined with free jejunal graft after total pharyngolaryngoesophagectomy. World J Surg 2017;41:2329-36. DOI PubMed

27. Ni S, Zhu Y, Li D, et al. Gastric pull-up reconstruction combined with free jejunal transfer (FJT) following total pharyngolaryngooesophagectomy (PLE). Int J Surg 2015;18:95-8. DOI PubMed

28. Sharma S, Gupta DK. Surgical techniques for esophageal replacement in children. Pediatr Surg Int 2017;33:527-50. DOI PubMed

29. Coevoet D, Van Daele E, Willaert W, et al. Quality of life of patients with a colonic interposition postoesophagectomy. Eur J Cardiothorac Surg 2019;55:1113-20. DOI PubMed

30. Koh HK, Tan NC, Tan BK, Ooi ASH. Comparison of outcomes of fasciocutaneous free flaps and jejunal free flaps in pharyngolaryngoesophageal reconstruction: a systematic review and meta-analysis. Ann Plast Surg 2019;82:646-52. DOI PubMed

31. Watanabe M, Baba Y, Yoshida N, et al. Modified gastric pull-up reconstructions following pharyngolaryngectomy with total esophagectomy. Dis Esophagus 2014;27:255-61. DOI PubMed

32. Marks SC, Steiger Z. Combined pectoralis flap and gastric pull-up for pharyngeal reconstruction. Head Neck 1997;19:134-6. DOI PubMed

33. Liu J, Zhang Y, Li Z, Liu S, Li H, Xu Z. Benefit of salvage total pharyngolaryngoesophagectomy for recurrent locally advanced head and neck cancer after radiotherapy. Radiat Oncol 2017;12:164. DOI PubMed PMC

34. Meulemans J, Couvreur F, Beckers E, et al. Oncologic and functional outcomes after primary and salvage laryngopharyngoesophagectomy with gastric pull-up reconstruction for locally advanced hypopharyngeal squamous cell carcinoma. Front Oncol 2019;9:735. DOI PubMed PMC

35. McLean JN, Nicholas C, Duggal P, et al. Surgical management of pharyngocutaneous fistula after total laryngectomy. Ann Plast Surg 2012;68:442-5. DOI PubMed

36. Ho CM, Lam KH, Wei WI, Yuen PW, Lam LK. Squamous cell carcinoma of the hypopharynx--analysis of treatment results. Head Neck 1993;15:405-12. DOI PubMed

37. Morita M, Saeki H, Ito S, et al. Technical improvement of total pharyngo-laryngo-esophagectomy for esophageal cancer and head and neck cancer. Ann Surg Oncol 2014;21:1671-7. DOI PubMed

38. Puttawibul P, Pornpatanarak C, Sangthong B, et al. Results of gastric pull-up reconstruction for pharyngolaryngo-oesophagectomy in advanced head and neck cancer and cervical oesophageal squamous cell carcinoma. Asian J Surg 2004;27:180-5. DOI PubMed

39. Okita Y, Kobayashi M, Araki T, et al. Impact of Surgical Infection Society/Infectious Disease Society of America-recommended antibiotics on postoperative intra-abdominal abscess with image-guided percutaneous abscess drainage following gastrointestinal surgery. Surg Today 2015;45:993-1000. DOI PubMed

40. Okita Y, Mohri Y, Kobayashi M, et al. Factors influencing the outcome of image-guided percutaneous drainage of intra-abdominal 
abscess after gastrointestinal surgery. Surg Today 2013;43:1095-102. DOI PubMed PMC

41. McNeeley MF, Vo NJ, Prabhu SJ, Vergnani J, Shaw DW. Percutaneous drainage of intra-abdominal abscess in children with perforated appendicitis. Pediatr Radiol 2012;42:805-12. DOI PubMed

42. Ballard DH, Erickson AEM, Ahuja C, Vea R, Sangster GP, D'Agostino HB. Percutaneous management of enterocutaneous fistulae and abscess-fistula complexes. Dig Dis Interv 2018;2:131-40. DOI PubMed PMC

43. Gedda S, van der Linden W. What makes the peritoneal drain work? Acta Chir Scand 1983;149:703-706. PubMed

44. Jiang T, Li J, Ren J. Continuous negative pressure drain is associated with better outcome: a randomized prospective trial in plastic surgery patients. Aesthetic Plast Surg 2019;43:91-7. DOI PubMed

45. Schintler M. Vacuum assisted closure system in the management of cervical anastomotic leakage after gastric pull-up. Interact Cardiovasc Thorac Surg 2004;3:92-4. DOI PubMed

46. Kim SI, Lim MC, Song YJ, Seo SS, Kang S, Park SY. Application of a subcutaneous negative pressure drain without subcutaneous suture: impact on wound healing in gynecologic surgery. Eur J Obstet Gynecol Reprod Biol 2014;173:94-100. DOI PubMed

47. FDA News Release. Patient-reported outcome measures: use in medical product development to support labeling claims. Available from: https://www.fda.gov/regulatory-information/search-fda-guidance-documents/patient-reported-outcome-measures-use-medicalproduct-development-support-labeling-claims [Last accessed on Dec 2009].

48. Wu TY, Chen CT, Huang YJ, Hou WH, Wang JD, Hsieh CL. Rasch analysis of the 9-item shared decision making questionnaire in women with breast cancer. Cancer Nurs 2019;42:E34-42. DOI PubMed

49. Yang SC, Lai WW, Hsu JC, Su WC, Wang JD. Comparative effectiveness and cost-effectiveness of three first-line EGFR-tyrosine kinase inhibitors: Analysis of real-world data in a tertiary hospital in Taiwan. PLoS One 2020;15:e231413. DOI PubMed PMC

50. Rogers S, Humphris G, Lowe D, Brown J, Vaughan E. The impact of surgery for oral cancer on quality of life as measured by the Medical Outcomes Short Form 36. Oral Oncology 1998;34:171-9. DOI PubMed 